\title{
Metformina versus insulina para el tratamiento de diabetes gestacional (1)
}

\author{
Rowan JA, Hague WM, Gao W, Battin MR, Moore MP; MiG Trial Investigators. N Engl J \\ Med 2008;358(19):2003-15.
}

Análisis crítico: Paula Vargas I. ${ }^{1}$, Jorge Carvajal C. ${ }^{1}$, PhD.

1 Unidad de Medicina Materno Fetal, Departamento de Obstetricia y Ginecología, Facultad de Medicina, Pontificia
Universidad Católica de Chile.

\section{RESUMEN (1)}

Introducción: Metformina es un tratamiento lógico para pacientes con diabetes gestacional, pero existe una deficiencia de estudios randomizados que demuestren su eficacia y seguridad. Métodos: Se randomizaron 751 mujeres con diagnóstico de diabetes gestacional entre $20-33$ semanas de gestación, se inició tratamiento abierto de metformina (agregando insulina cuando fuera necesario) e insulina. El resultado primario a medir fue compuesto en relación a hipoglicemia neonatal, distrés respiratorio, trauma en el parto, Apgar a los 5 minutos $<7$, necesidad de fototerapia y prematurez. El estudio fue diseñado para lograr un 33\% de aumento (de 30 a $40 \%$ ) en este resultado compuesto en pacientes tratadas con metformina vs pacientes que recibieron insulina. Resultados secundarios incluyeron medidas antropométricas neonatales, control de glicemia materna, complicaciones hipertensivas maternas, tolerancia a la glucosa postparto y aceptación del tratamiento. Resultados: De las 363 mujeres asignadas a metformina, 92,6\% mantuvo el tratamiento hasta el parto, 46,3\% debió agregar insulina como suplemento. La tasa de resultado primario compuesto fue $32 \%$ en el grupo asignado a metformina y $32,2 \%$ en el grupo de insulina (riesgo relativo: 1,0; IC 95\%, 0,9-1,1). Más mujeres en el grupo de metformina que el de insulina declararon que si tuvieran que repetir seguirían con el tratamiento asignado ( $76,6 \%$ vs $27,2 \%$; $p<0,001)$. La tasa de resultados secundarios no tuvo diferencia significativa entre los grupos. No hubo efectos adversos serios asociados con uso de metformi- na. Conclusiones: En mujeres con diabetes gestacional, el uso de metformina (sola o asociada a insulina) comparado con insulina, no se asocia a aumento de complicaciones perinatales. Las mujeres prefieren usar metformina que insulina como tratamiento.

\section{ANÁLISIS DE LA INVESTIGACIÓN}

\section{A. Relevancia clínica de la investigación}

En el manejo de la diabetes mellitus gestacional con mal control metabólico en respuesta a la dieta: ¿es posible utilizar metformina en lugar de insulina para optimizar el control metabólico y mejorar el resultado perinatal?

Escenario clínico: La diabetes gestacional se presenta en una proporción variable de las embarazadas, con un rango entre 3 y $10 \%$, dependiendo de la población estudiada, el tipo de screening utilizado y el tipo de medio diagnóstico (2). Si bien sus consecuencias son moderadas, la diabetes gestacional significa un mayor riesgo materno y perinatal, y un incremento en el riesgo de presentar diabetes mellitus tipo 2 en años posteriores. La mayoría de las mujeres logra un buen control metabólico mediante ajuste dietario, sin embargo, cerca de un $5 \%$ de las mujeres requiere un tratamiento adicional, siendo el estándar el uso de insulina. De modo más reciente, se ha demostrado la seguridad y factibilidad del uso de hipoglicemiantes orales en este escenario, principalmente gliburida y en menor proporción metformina (3). 


\section{B. El estudio (1)}

Diseño: estudio prospectivo randomizado abierto, efectuado en 10 centros obstétricos urbanos de Nueva Zelanda y Australia.

Pacientes: 751 mujeres embarazadas entre 20-33 semanas, embarazos únicos, con diagnóstico de diabetes gestacional que no respondieron a dieta y que, en su manejo estándar, requerirían tratamiento con insulina. Se excluyeron pacientes con diabetes pregestacional, contraindicación de uso de metformina, hipertensión del embarazo, preeclampsia, restricción de crecimiento fetal, rotura de membranas y malformaciones fetales.

Intervención: 363 pacientes asignadas a metformina. Recibieron $500 \mathrm{mg} 1 \mathrm{o}$ dos veces al día con las comidas. Se ajustó la dosis luego de 1 o 2 semanas, hasta alcanzar el objetivo terapéutico o una dosis máxima de $2500 \mathrm{mg}$. El objetivo terapéutico fue lograr glicemia de ayuno $<99 \mathrm{mg} / \mathrm{dl}$ y post prandial $(2$ h) <126 mg/dl. Comparación: 370 pacientes asignadas a insulina, la cual fue administrada de acuerdo al manejo estándar de cada centro.

Resultados: Resultado primario: morbilidad compuesta neonatal que incluye hipoglicemia neonatal, distrés respiratorio, trauma en el parto, Apgar a los 5 minutos $<7$, necesidad de fototerapia y prematurez. Resultados secundarios: medidas antroprométricas neonatales, control de glicemia materna, complicaciones hipertensivas maternas, tolerancia a la glucosa postparto y aceptación del tratamiento. No hubo diferencias en el resultado primario ni en los resultados secundarios (Tabla I).

\section{Tabla I}

\section{RESULTADO PRIMARIO GRUPO METFORMINA} VS INSULINA

\begin{tabular}{lcccc}
\hline & $\begin{array}{c}\text { Metformina } \\
(\mathrm{n}=363)\end{array}$ & $\begin{array}{c}\text { Insulina } \\
(\mathrm{n}=370)\end{array}$ & $\begin{array}{c}\mathrm{RR} \\
(\mathrm{IC} 95 \%)\end{array}$ & Valor $\mathrm{p}$ \\
\hline Resultado & 116 & 119 & 1,0 & 0,95 \\
compuesto & $(32 \%)$ & $(32,2 \%)$ & $(0,9-1,1)$ & \\
\hline
\end{tabular}

\section{Análisis crítico}

Validez interna: Se trata de un estudio randomizado, con ocultamiento de la secuencia de asignación. Se efectuó randomización computacional, estratificando por centros y edad gestacional. Los grupos fueron similares respecto a variables pronósticas conocidas, lo que demuestra la calidad de la randomización. Se calculó de modo correcto el tamaño muestral y se reclutaron los pacientes necesarios. El seguimiento fue apropiado (98\%) y el análisis se efectuó según intención de tratar. El estudio fue abierto (no ciego), pero se indica quien adjudicó los eventos, y fue ciego a la intervención quien analizó los datos.

Comentarios: El estudio es adecuado a la pregunta y su validez interna fue bien cautelada. No fue un estudio ciego, lo que introduce una posibilidad de sesgo, sin embargo, es la única manera de hacer posible un análisis respecto de la aceptabilidad del tratamiento. Considerando que el resultado principal fue asignado por un investigador ciego a la intervención, consideramos que la falta de ciego del paciente y el tratante no es un problema mayor.

Existen dos aspectos importantes a considerar en este estudio: se trata de un estudio de "no inferioridad" y se usó un resultado primario compuesto. Los estudios de no inferioridad son de utilidad en la introducción de un nuevo tratamiento, en que no es ético efectuar un estudio versus placebo. Los estudios diseñados para demostrar no inferioridad requieren una población de referencia apropiada, un manejo activo del control clínico y las dosis del medicamento, un margen apropiado de no inferioridad que sea clínicamente relevante y estadísticamente justificable, un alto nivel de adherencia al tratamiento y un adecuado poder estadístico. Si se cumplen estos parámetros, es posible asegurar que el nuevo tratamiento no es inferior que el estándar, y por lo tanto es efectivo (4). Estimamos que este estudio cumple con los requisitos descritos y es efectivo en demostrar la no inferioridad de la metformina, sin embargo, el $46,3 \%$ de las pacientes asignadas a metformina requirieron suplemento con insulina. Estas pacientes eran más obesas y con peor control metabólico al ingreso al estudio, sugiriendo que en este subgrupo deben tenerse consideraciones especiales.

El uso de un resultado compuesto es muy frecuente en obstetricia, con la idea de evitar la necesidad de grandes tamaños maestrales, sin embargo, su uso requiere consideraciones (5). Un resultado compuesto es aceptable si todos sus componentes tiene frecuencia similar y si existe lógica en combinarlos. Creemos que este estudio cumple con los criterios necesarios para un resultado combinado aceptable; no muestra diferencias respecto del resultado primario ni secundario, por lo que ambos tratamientos serían comparables. Observamos, que el resultado primario no considera el peso de nacimiento o la probabilidad de crecimiento mayor a percentil 90 , siendo este un problema mayor del manejo de la diabetes gestacional.

Conclusión: Creemos que el estudio es apropiado y que sus resultados son aplicables a nuestra población por la facilidad de administración de la 
droga (oral versus subcutáneo), la poca frecuencia de efectos adversos y el fácil seguimiento de las pacientes en su control prenatal regular.

\section{BIBLIOGRAFÍA}

1. Rowan JA, Hague WM, Gao W, Battin MR, Moore MP; MiG Trial Investigators. Metformin versus insulin for the treatment of gestational diabetes. $\mathrm{N}$ Engl $\mathrm{J}$ Med 2008;358(19):2003-15.

2. Hollander MH, Paarlberg KM, Huisjes AJ. Gestational diabetes: a review of the current literature and guidelines. Obstet Gynecol Surv 2007;62(2):125-36.

3. Coustan DR. Pharmacological management of gestational diabetes: an overview. Diabetes Care 2007; 30 Suppl 2:S206-8.

4. Kaul S, Diamond GA. Good enough: a primer on the analysis and interpretation of noninferiority trials. Ann Intern Med 2006;145(1):62-9.

5. Ross S. Composite outcomes in randomized clinical trials: arguments for and against. Am J Obstet Gynecol 2007;196(2):119.e1-6. 